\title{
Presión poblacional y sistema de oferta alimentaria en los países en desarrollo*
}

\author{
John Bongaarts**
}

En el presente trabajo se analizan las tendencias de la producción agricola en los paises en desarrollo entre 1962 y 1989, con el fin de obtener estimaciones de las contribuciones que ciertos elementos han hecho a la pasada expansión de la oferta alimentaria, como son: el aumento en el uso de tierras, la frecuencia de cosecha, el volumen de las cosechas y las importaciones.

Los países con altas o bajas densidades de población respondieron de muy diversa manera a la creciente demanda de alimentos. Durante los próximos cincuenta años, el rápido crecimiento poblacional y el continuo mejoramiento en la cantidad y calidad de las dietas tendrán como resultado un gran aumento (quizá hasta el triple) en la demanda de alimentos. Si bien no se prevé una constante escasez global, lo cierto es que algunos problemas - como la degradación de los recursos ambientales, la producción de alimentos en los países más densamente poblados y más pobres, o la desnutrición-requieren de una atención concertada.

El que la producción de alimentos pueda -o no- mantener el ritmo de la demanda de dietas mejoradas para una población mundial en rápido crecimiento, es un hecho que se ha debatido con vehemencia desde que fue planteado por Malthus hace dos siglos (Malthus, 1959). Aunque una gran parte de la humanidad ha experimentado un mejoramiento en su dieta durante el último siglo, las opiniones de los expertos sobre las perspectivas para el siguiente difieren de manera tan tajante como antes. ${ }^{1}$ Los pesimistas advierten los muchos signos de desgaste ambiental, así como las crecientes dificultades para la expansión de las tierras agrícolas, para el suministro de agua y de cosechas, y para el control de plagas (Brown y Kane, 1994; Ehrlich y Ehrlich, 1990; Ehrlich, Ehrlich y Daily, 1993). Desde su punto de vista no es factible lograr una gran expansión de la producción agrícola, e incluso dudan de que en algunos países se puedan mantener los ac-

\footnotetext{
* El presente artículo fue traducido del inglés, con el permiso de The Population Council, de "Population Pressure and the Food Supply System in the Developing World", publicado en Population and Developing Review, vol, 22, núm. 3, pp. 483-503.

** El autor agradece los comentarios y sugerencias a borradores anteriores de este artículo hechos por Nikos Alexandratos, Joel Cohen y Tim Dyson.

${ }^{1}$ Respecto de las revisiones y comentarios recientes sobre las perspectivas de alimentar a una creciente población mundial, véanse Agcaoili y Rosegrant (1995); Alexandratos (1995); Cohen (1995); Dyson (1996); Mitchell e Ingco (1995); y Smil (1994).
} 
tuales rangos de producción. En contraste, los optimistas destacan que en la actualidad los rendimientos de las cosechas son relativamente ba:jos, existen ineficiencias en la cadena de producción y consumo de alimentos, y amplias reservas de tierras potencialmente cultivables en muchos países en desarrollo (Heilig, 1994; Johnson, 1984; Simon, 1981; Smil, 1987 y 1994). Asimismo sostienen que algunas medidas, como políticas ambientales más profundas, mayor aplicación de tecnología de la llamada revolución verde, reducción de las ineficiencias, el mejoramiento de la infraestructura rural y mayor inversión en recursos humanos e investigación harán posible obtener cosechas mucho mayores. Además, ellos no prevén la existencia de restricciones ambientales insalvables.

A pesar de este desacuerdo respecto de las dificultades futuras, hay una disputa menor sobre las tendencias en la demanda de alimentos para las décadas venideras. Naciones Unidas (1995) calcula que habrá una población mundial de 9800 millones para el año 2050 , contra los 5800 millones actuales. Por ello, y con el fin de que las mejoras en la dieta se incrementen tal como se espera, en muchos de los países en desarrollo se necesitará una enorme expansión de la producción agrícola en las próximas décadas; satisfacer esta demanda será uno de los mayores retos de la humanidad.

Es muy probable que el futuro no sea ni tan problemático como sostienen los pesimistas, ni tan desprovisto de dolor como prevén algunos optimistas. Entonces, ¿cuáles son las tendencias futuras más plausibles? Este estudio pretende arrojar luz sobre esta cuestión examinando las tendencias del sistema de oferta alimentaria entre 1962 y $1989,{ }^{2}$ y se concentra en los países en desarrollo, donde los problemas agrícolas actuales y futuros son más serios. El análisis se basa en las estadísticas de la Organización de las Naciones Unidas para la Agricultura y la Alimentación (FAO), que cubren los distintos aspectos del sistema de oferta alimentaria en los 90 países en desarrollo, y para los cuales está disponible la información necesaria (FAO, 1990 y 1992; Alexandratos, 1995). Se presta particular atención a la manera en que los países con diferentes densidades de población han alimentado a sus crecientes poblaciones.

${ }^{2}$ A lo largo de este trabajo, los promedios de 1962 y 1989 representan promedios trianuales, es decir, 1961-1963 y 1988-1990. 


\section{Marco analítico}

Para analizar los complejos procesos por los cuales la tierra potencialmente cultivable se usa para producir alimentos, y con el propósito de medir, para cada país, las tendencias de la tierra y otros recursos necesarios para cultivar, me baso en un conjunto de variables interrelacionadas que se enlistan en las figuras 1 y 2 y en el cuadro 1 , mismas que se describen a continuación. ${ }^{3}$

FIGURA 1

Variables clave en el análisis del sistema de oferta alimentaria

Tierra potencialmente cultivable

$$
\begin{gathered}
\text { Tierra cultivable } \\
\text { Área cultivada } \\
\mathrm{F}_{\mathrm{f}} \downarrow
\end{gathered}
$$

Área dedicada a los cultivos alimenticios

$$
F_{y} \downarrow
$$

Producción interna de cultivos Otras fuentes de forraje

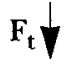

Uso interno de las cosechas

$$
F_{d} \downarrow
$$

Consumo humano directo<smiles></smiles>

Consumo humano total

Nota: todas las variables que no se refieren a áreas se miden en términos de toneladas de grano equivalente (TGE).

${ }^{3}$ Una discusión detallada respecto a estas variables y procesos aparece en Alexandratos (1995). 
FIGURA 2

Variables clave en el sistema de oferta alimentaria

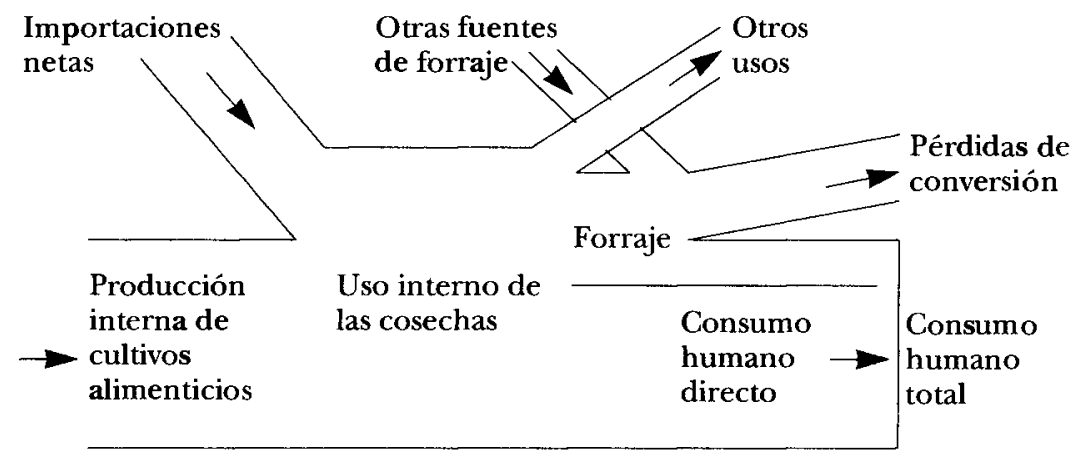

CUADRO 1

Estimaciones de uso de tierra y de producción y consumo de cultivos alimenticios para 90 países en desarrollo en 1989

\begin{tabular}{lcc}
\hline & $\begin{array}{c}\text { Total } \\
\text { (millones de ha) }\end{array}$ & $\begin{array}{c}\text { Per cápita } \\
(\text { ha })\end{array}$ \\
\hline Tierra & 7325 & 1.87 \\
Tierra potencialmente cultivable & 2667 & 0.68 \\
Tierra cultivable & 853 & 0.22 \\
Área cultivada & 740 & 0.19 \\
Área dedicada a los cultivos de alimentos & 696 & 0.18 \\
& & \\
Producción interna de cultivos & $($ TGE) & $($ TGE) \\
Uso interno de las cosechas & 1560 & 0.40 \\
Consumo humano directo & 1610 & 0.41 \\
Consumo humano de alimentos & 1190 & 0.30 \\
& 1320 & 0.34
\end{tabular}

a toneladas de grano equivalente.

Nota: la población total de los 90 países es de 3922 millones, y el área total de los países en desarrollo es de 7587 millones de hectáreas. 


\section{Tierra potencialmente cultivable}

La FAo estima que la cantidad de suelo adecuado para los cultivos de temporal en los países en desarrollo incluidos en este estudio (con excepción de China) es de 2600 millones de hectáreas. ${ }^{4} \mathrm{Si}$ a esto se suma una estimación gruesa de los 100 millones que corresponden a China se obtiene un total de 2700 millones de hectáreas, lo cual significó 0.68 ha per cápita en 1989, para una población de 3920 millones. ${ }^{5}$ El suelo restante -cerca de $64 \%$ del total- está constituido por desiertos, montañas y tierra con otro tipo de restricciones severas.

\section{Tierra cultivable}

La cantidad de tierra que de hecho se cultiva es una fracción del área potencialmente cultivable (para los países en desarrollo, la proporción cultivada actualmente es 0.32 ); gran parte del área que no se utiliza está cubierta por bosques y se estima que una pequeña aunque creciente fracción ( $3 \%$ en promedio) está bajo asentamientos humanos o infraestructura. Estas "reservas" tienen regularmente un limitado atractivo para la agricultura en los países donde se localizan, debido a la falta de acceso a los mercados, a la calidad relativamente baja de la tierra y a la escasez de recursos hidrológicos.

\section{Área cultivada}

La frecuencia con que se cosecha la tierra cultivable varía ampliamente entre los distintos países. En algunos lugares, particularmente en la región de África subsahariana, una gran proporción de tierra cultivable se deja en barbecho a veces durante un año o más, mientras que en otros sitios se obtiene más de una cosecha al año si hay disponibili-

\footnotetext{
${ }^{4}$ Los cálculos de la fao sobre la tierra potencialmente cultivable incluyen $36 \mathrm{mi}$ llones de hectáreas de desierto sin potencial de lluvias, que se han convertido al uso agrícola por medio de la irrigación.

${ }^{5}$ Los 90 países incluidos en este estudio reúnen $98 \%$ de la población de todos los países en desarrollo (la falta de datos impidió la inclusión de los países restantes). La cantidad de tierra potencialmente cultivable de China se estimó usando la superficie cultivable de 96 millones de hectáreas consignada en la base de datos Agrostat (FAo, 1992) asumiendo que la proporción de tierra cultivada es de 90 por ciento.
} 
dad de agua y el clima es favorable. Las cosechas múltiples son posibles en gran parte del trópico, especialmente donde se dispone de irrigación, ya que las temperaturas y la época de cultivo son relativamente constantes durante todo el año. Así, en un país el área realmente cosechada puede ser mayor o menor al área cultivable. La frecuencia de cosecha se define como el número promedio de cosechas obtenidas cada año en tierra cultivable; en 1989, dicha frecuencia fue equivalente a 0.87 en los países en desarrollo. ${ }^{6}$ El área cultivada ( 740 millones de hectáreas) equivale a la frecuencia de cosecha multiplicada por la tierra cultivable (853 millones de hectáreas).

\section{Área dedicada a los cultivos alimenticios}

En muchos países una proporción significativa del área cultivada produce cultivos que no son alimenticios, como el café, té o fibras. En 1989, en promedio, 94\% del área cultivada se utilizó para cultivos alimenticios.

\section{Producción cosechada}

La cantidad de un cultivo alimenticio producida en una sola cosecha, en una hectárea de tierra, equivale al rendimiento de esa cosecha. El rendimiento varía de un cultivo a otro y depende de las condiciones de la tierra y el clima, la variedad de la semilla, la disponibilidad de agua y el uso de fertilizantes, pesticidas y otro tipo de tecnología. Para estimar el total de la producción cosechada, los cultivos que no son cereales (como azúcar, semillas oleaginosas/aceites vegetales, tubérculos, leguminosas, frutas y verduras) se convierten a sus equivalentes en cereal según su contenido calórico. En 1989 la producción alimentaria total en los países en desarrollo fue de 1560 millones de TGE (toneladas de grano equivalente), y fue obtenida de 696 millones de hectáreas de área cultivada. ${ }^{7}$ Por lo tanto, el rendimiento promedio por cosecha fue de $2.2 \mathrm{TGE} /$ hectárea.

${ }^{6}$ Alexandratos (1995) utiliza el término "intensidad de cosecha", el cual considera el área cosechada como porcentaje del área cultivable; de este modo, la intensidad de cosecha equivale a la frecuencia de cosecha multiplicada por 100.

${ }^{7}$ La producción estimada incluye arroz en términos del equivalente sin cáscara. 
Uso interno de las cosechas

Las importaciones y exportaciones pueden aumentar o reducir la cantidad de cosechas alimenticias disponibles para el uso interno. La proporción del uso interno respecto de la producción interna, calculada en términos de TGE, se define como el multiplicador de comercio. Este coeficiente fue de 1.03 en 1989 en los países en desarrollo, lo cual implica que la importación neta correspondió a $3 \%$ de la producción interna.

\section{Consumo humano directo}

El principal uso interno de los cultivos alimenticios se dedica al consumo humano directo que, en promedio, sumó $74 \%$ del total en los países en desarrollo. Otros usos para este tipo de cultivos son: forraje, simiente y usos industriales no alimenticios; y, por supuesto, parte de estos cultivos se pierde entre la granja y el hogar. Todas las estimaciones de la FAo referentes al consumo humano se basan en las cantidades de alimento que llegan a los hogares; en ellos ocurren pérdidas adicionales, pero faltan estadísticas al respecto. El desperdicio de alimentos en el hogar es probablemente mayor en los países de altos ingresos; de esta manera las diferencias en el consumo humano real entre los países de altos y bajos ingresos quizá sean menores de lo que sugieren las estadísticas de la fao (Smil, 1987).

\section{Consumo total de alimentos}

La mayoría de las dietas contienen productos tanto vegetales como animales. Los segundos se componen casi íntegramente de productos ganaderos, como carne y lácteos. ${ }^{8}$ En los países en desarrollo el ganado se ha alimentado tradicionalmente con pastos y productos secundarios de los cultivos, pero está aumentando la proporción de forraje derivado de cultivos -como los cereales- que compite con el consumo humano. En 1989 el consumo calórico promedio para los países en desarrollo se estimó en 2472 calorías diarias por persona, y

${ }^{8}$ El pescado y otros mariscos dan cuenta de una fracción de $1 \%$ de la dieta promedio del mundo en desarrollo. 
9.2\% de éste se derivó de los productos animales. ${ }^{9}$ La proporción del consumo calórico total respecto del contenido calórico de origen vegetal, llamado multiplicador de producto animal, fue de 1.11 en 1989.

La cadena de variables enlistadas en la figura 1 produce una serie de coeficientes cuando cada variable se divide entre la anterior. Los siete factores que se enumeran a continuación cuantifican los aspectos clave de la operación del sistema de oferta alimentaria (las cifras entre paréntesis indican el valor numérico estimado para cada factor en 1989, tomando como base un total de 90 países en desarrollo):

$F_{\mathbf{u}}=$ proporción de tierra potencialmente cultivable que se cultiva $(0.32)$

$F_{c}=$ frecuencia de cosecha $(0.87)$

$F_{\mathrm{f}}=$ proporción del área cultivada utilizada para la producción de cosechas alimenticias $(0.94)$

$\mathrm{F}_{\mathrm{y}}=$ rendimiento promedio de las cosechas, en TGE (2.24)

$\mathrm{F}_{\mathrm{t}}=$ multiplicador de comercio (1.03)

$\mathrm{F}_{\mathrm{d}}=$ proporción de uso interno para consumo humano directo $(0.74)$

$\mathrm{F}_{\mathrm{a}}=$ multiplicador de producto animal (1.11)

El producto de estos factores y la cantidad de tierra potencialmente cultivable $(L)$ equivale a la oferta de alimento disponible para el consumo humano directo. La demanda de alimento equivale al producto del tamaño de la población $(P)$, y al consumo alimenticio per cápita expresado en toneladas de grano equivalente (TGE) por año $(c)$, de modo que:

$$
\begin{aligned}
& P c=\mathrm{F}_{\mathrm{u}} \mathrm{F}_{\mathrm{c}} \mathrm{F}_{\mathrm{f}} \mathrm{F}_{\mathrm{y}} \mathrm{F}_{\mathrm{t}} \mathrm{F}_{\mathrm{d}} \mathrm{F}_{\mathrm{a}} L \\
& \text { (demanda) } \\
& \text { (oferta) }
\end{aligned}
$$

Como ya se mencionó, en 1989 la tierra potencialmente cultivable era de 2670 millones de hectáreas. Al multiplicar por $0.50 \mathrm{el}$ producto de los siete factores -es decir, $0.32 \times 0.87 \times 0.94 \times 2.24 \times 1.03 \times$ $0.74 \times 1.11-\mathrm{da}$ como resultado un consumo total de 1320 millones de TGE por año o 0.34 TGE por año per cápita, para una población de 3920 millones, en los países en desarrollo.

$\mathrm{El}$ análisis propuesto en este trabajo está basado en estimaciones de tendencias pasadas de los factores de la oferta en los países en desa-

\footnotetext{
${ }^{9}$ De acuerdo con la práctica de la FAo, la estimación del consumo calórico diario per cápita se participa con una precisión de cuatro cifras significativas. En realidad, para casi todos los países, cuando mucho las dos primeras cifras de estas estimaciones son significativas.
} 
rrollo que forman parte de este estudio. Qué tanto cambia cada factor en respuesta a la creciente demanda de alimento, está determinado por un complejo conjunto de condiciones económicas, sociales, políticas y a mbientales. Los incrementos generalmente serán mayores en los factores cuyos aumentos marginales sean menos costosos de lograr. Además, expandir la oferta de alimentos en un ambiente con recursos naturales fijos implica aumentar los costos marginales, lo cual, manteniendo constante todo lo demás, provoca incrementos en los precios de los alimentos. Tales aumentos se pueden evitar $-y$ se han evitado en el pasado- si se mejora y amplía la productividad por medio de cambios en el uso de tecnología; de hecho, los precios de los alimentos en el mercado mundial se han reducido considerablemente en las últimas décadas (Banco Mundial, 1992). Las importaciones también pueden reducir la presión sobre los precios en países que imponen severas restricciones a los recursos agrícolas.

\section{Tendencias de la oferta, 1962-1989}

Entre 1962 y 1989 la población de los países en desarrollo aumentó $84 \%$, y el consumo per cápita $27 \%$; de esta manera, el consumo total de alimentos subió $134 \%$. Esta expansión se logró por medio de los aumentos en sólo algunos factores que determinan la oferta. Como se muestra en la gráfica 1, la mayoría de los factores cambió sólo ligeramente, aunque la proporción de la tierra cultivada $(+18 \%)$, la frecuencia de cosecha $(+11 \%)$ y el rendimiento de la cosecha $(+75 \%)$ aumentaron sustancialmente. El incremento en el rendimiento promedio por cosecha fue, por mucho, el cambio más importante -como se esperaba- motivado por la difusión de la revolución verde en gran parte de los países en desarrollo durante las últimas tres décadas.

Los resultados de la gráfica 1 corresponden a la combinación de los 90 países en desarrollo, esto es, la población, tierra, producción de alimentos, consumo y demás, se calculan sumando las estimaciones correspondientes de los países considerados individualmente. Los promedios resultantes están ponderados por el tamaño relativo de cada país, lo cual implica que las experiencias de los países más grandes (en particular China e India) influyen fuertemente en los resultados, mientras que las de los países pequeños tienen un impacto menor. Otra posibilidad de obtener el promedio general es tratar a cada país como una unidad de análisis y promediar las estimaciones 
GRÁFICA 1

Cambio porcentual en población, consumo alimenticio per cápita y factores de la oferta alimentaria en los países en desarrollo, 1962-1989

(promedios ponderados)

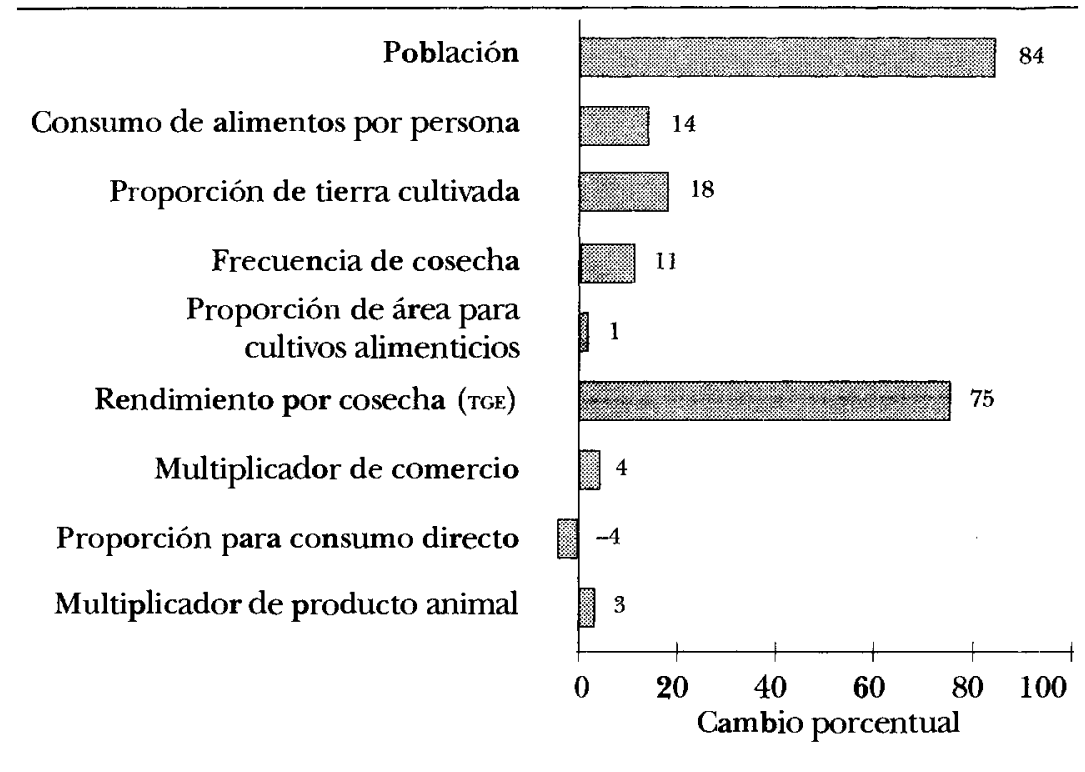

de los factores para los distintos países. En un promedio simple (no ponderado) la experiencia de cada país es igualmente importante. Los cambios en los promedios de los factores para estos países se presentan en la gráfica 2 y el cuadro 2 . Si bien estas tendencias son, a grandes rasgos, similares para la mayoría de los factores de la oferta -sobre todo si se comparan con los promedios ponderados de la gráfica 1-, son completamente diferentes en lo que se refiere al rendimiento por cosecha (aumento de 41 en lugar de $75 \%$ ) y al comercio de alimentos ( 35 en lugar de $4 \%$ ). La diferencia entre estos dos tipos de promedio se explica por el hecho de que China, India y varios otros grandes países comercian poco, aunque han logrado relativamente más éxito en aumentar los rendimientos. En lo que resta de este artículo se usarán promedios no ponderados, ya que su objetivo es explicar cómo los países se adaptan a la presión poblacional. 


\section{GRÁFICA 2}

Cambio porcentual en población, consumo alimenticio per cápita y factores de la oferta alimentaria en los países en desarrollo, 1962-1989 (promedios no ponderados)

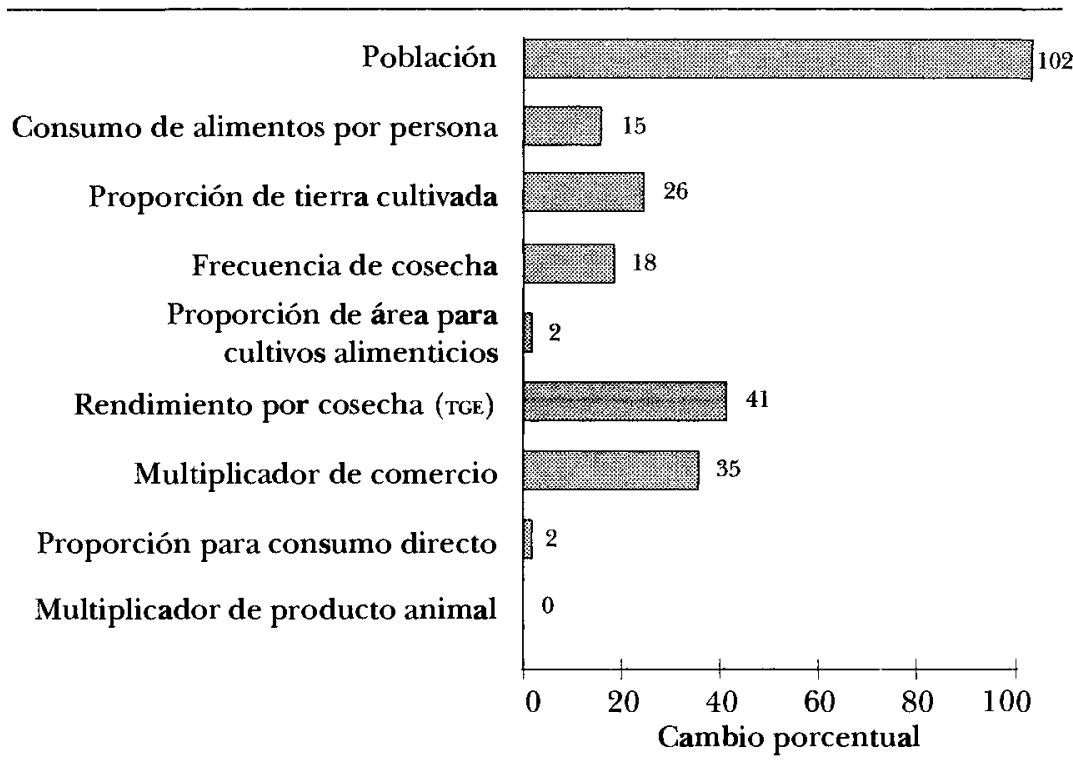

CUADRO 2

Estimaciones de los factores de la oferta alimentaria para 90 países en desarrollo

\begin{tabular}{|c|c|c|c|c|}
\hline \multirow[b]{2}{*}{ Factor de la oferta } & \multicolumn{2}{|c|}{ Promedio ponderado } & \multicolumn{2}{|c|}{$\begin{array}{c}\text { Promedio } \\
\text { no ponderado }\end{array}$} \\
\hline & 1962 & 1989 & 1962 & 1989 \\
\hline Proporción de tierra cultivada & 0.27 & 0.32 & 0.40 & 0.48 \\
\hline Frecuencia de cosecha & 0.78 & 0.87 & 0.67 & 0.73 \\
\hline $\begin{array}{l}\text { Proporción de tierra para } \\
\text { cultivos alimenticios }\end{array}$ & 0.93 & 0.94 & 0.92 & 0.94 \\
\hline Rendimiento por cosecha (TGE) & 1.28 & 2.24 & 1.52 & 1.95 \\
\hline Multiplicador de comercio & 0.99 & 1.03 & 1.04 & 1.41 \\
\hline Proporción para consumo directo & 0.78 & 0.74 & 0.78 & 0.78 \\
\hline Multiplicador de producto animal & 1.08 & 1.11 & 1.12 & 1.12 \\
\hline
\end{tabular}


Densidad poblacional y el sistema de oferta alimentaria

La densidad poblacional, definida en este análisis como el número de personas por hectárea de tierra potencialmente cultivable, varía mucho entre los distintos países. En 1989 el país en desarrollo con mayor densidad era Egipto, con 19.2 personas por hectárea; ${ }^{10}$ en el otro extremo del espectro se localizó la República Centroafricana, con sólo 0.07 personas por hectárea. Para facilitar la presentación de los siguientes hallazgos, los países que comprenden este estudio se han dividido, de acuerdo con su densidad, en tres grupos de 30 países cada uno (cuadro 3). Los grupos de densidad alta, media y baja promediaron, respectivamente, $6.6,1.3$ y 0.3 personas por hectárea de tierra potencialmente cultivable. La parte superior del cuadro 4 presenta los promedios de los siete factores, así como los del consumo calórico per cápita para los distintos grupos en 1989 de acuerdo con las estimaciones de la FAO (1992). Las diferencias entre los grupos de alta y baja densidad poblacional fueron mayores según la proporción de tierra cultivada $(0.80$ contra 0.18$)$ y en la frecuencia de cosecha ( 1.01 contra 0.48 ). La diferencia es pequeña, aunque sustancial, tanto para el rendimiento por cosecha (2.40 contra $1.58 \mathrm{TGE})$, como para el multiplicador de comercio (1.73 contra 1.17), mientras que las diferencias en los demás factores fueron muy pequeñas. Sorprende que el consumo calórico haya sido significativamente mayor en el grupo de alta que en el de baja densidad ( 2477 contra 2270 calorías diarias por persona). En otras palabras, a pesar de contar con 22 veces más tierra ootencialmente cultivable por persona, los países con baja densidad poblacional tuvieron en promedio una dieta con menos calorías que los países con alta densidad. Una comparación de los porcentajes por los cuales los factores del grupo de alta densidad excedieron a los de baja (gráfica 3) muestra que este resultado se logró principalmente porque se utilizó una cantidad mayor de tierra potencialmente cultivable $(+350 \%)$, y hubo una mayor frecuencia de cosecha $(+109 \%)$. No obstante, la mayor importación de alimentos $(+48 \%)$ y los mayores rendimientos por cosecha $(+51 \%)$, desempeñaron también un papel significativo.

${ }^{10}$ Hong Kong y Singapur están más densamente poblados, pero no se incluyeron en el conjunto de los 90 países en desarrollo de este estudio. 
CUADRO 3

Países en desarrollo incluidos en este estudio, clasificados en tres grupos de 30 países cada uno, de acuerdo con su densidad poblacional en 1989

\begin{tabular}{|c|c|c|c|c|c|}
\hline \multicolumn{6}{|c|}{$\begin{array}{l}\text { Densidad poblacional en } 1989 \\
\text { (personas por ha de tierra potencialmente cultivable) }\end{array}$} \\
\hline \multicolumn{2}{|l|}{$\begin{array}{l}\text { Alta } \\
(6.6)\end{array}$} & \multicolumn{2}{|l|}{$\begin{array}{c}\text { Media } \\
(1.3)\end{array}$} & \multicolumn{2}{|l|}{$\begin{array}{l}\text { Baja } \\
(0.3)\end{array}$} \\
\hline Egipto & 19.19 & Irak & 2.23 & Senegal & 0.54 \\
\hline Mauricio & 15.99 & Siria & 2.23 & Costa de Marfil & 0.54 \\
\hline Arabia Saudita & 13.59 & Marruecos & 2.06 & Colombia & 0.52 \\
\hline Yemen & 13.11 & Tailandia & 2.05 & Benin & 0.50 \\
\hline Bangladesh & 11.88 & Libia & 1.83 & Tanzania & 0.48 \\
\hline China & 10.47 & Turquía & 1.82 & Burkina Faso & 0.47 \\
\hline Corea, Rep. de & 10.10 & Malasia & 1.71 & Liberia & 0.44 \\
\hline Ruanda & 9.21 & Lesotho & 1.63 & Zimbabwe & 0.43 \\
\hline Jordania & 7.44 & Nigeria & 1.62 & Venezuela & 0.42 \\
\hline Pakistán & 6.51 & Chile & 1.56 & Guinea & 0.42 \\
\hline Haití & 5.67 & Guatemala & 1.46 & Perú & 0.41 \\
\hline Líbano & 5.36 & México & 1.46 & Argentina & 0.37 \\
\hline Afganistán & 4.87 & Malawi & 1.41 & Camerún & 0.36 \\
\hline Sri Lanka & 4.77 & Cuba & 1.38 & Mali & 0.34 \\
\hline Somalia & 4.61 & Etiopía & 1.34 & Madagascar & 0.31 \\
\hline Jamaica & 4.59 & Uganda & 1.32 & Sudán & 0.30 \\
\hline Vietnam & 4.56 & Birmania & 1.29 & Mozambique & 0.28 \\
\hline Trinidad y Tobago & 4.44 & Gambia & 1.27 & Brasil & 0.25 \\
\hline Irán & 4.43 & Costa Rica & 1.09 & Uruguay & 0.24 \\
\hline India & 4.37 & Honduras & 1.05 & Zaire & 0.20 \\
\hline Corea, RDP de & 4.29 & Sierra Leona & 1.04 & Paraguay & 0.19 \\
\hline Burundi & 4.15 & Ghana & 0.92 & Chad & 0.18 \\
\hline El Salvador & 4.02 & Togo & 0.85 & Botswana & 0.18 \\
\hline Filipinas & 3.75 & Ecuador & 0,79 & Zambia & 0.14 \\
\hline Nepal & 3.50 & Camboya & 0.76 & Bolivia & 0.13 \\
\hline Indonesia & 3.09 & Panamá & 0.66 & Angola & 0.11 \\
\hline Túnez & 2.83 & Mauritania & 0.64 & Congo & 0.09 \\
\hline Rep. Dominicana & 2.69 & Nigeria & 0.64 & Guyana & 0.08 \\
\hline Kenya & 2.41 & Laos & 0.59 & Gabón & 0.07 \\
\hline Argelia & 2.26 & Nicaragua & 0.57 & Rep. Centroafricana & 0.07 \\
\hline
\end{tabular}

Nota: Los países están enlistados en orden decreciente de acuerdo con su densidad poblacional.

Fuentes: Alexandratos (1995), fao (1992); véanse también las notas 4 y 5. 


\section{GRÁFICA 3}

Diferencia porcentual entre los factores de la oferta alimentaria en países con alta y baja densidad, 1989

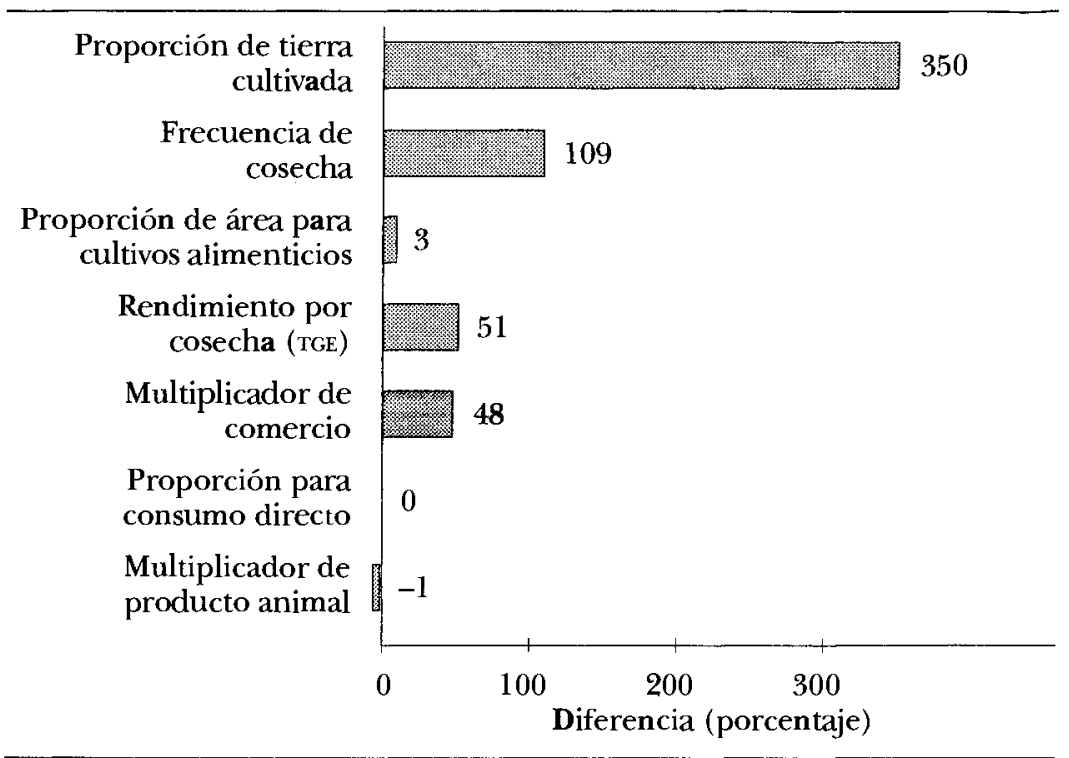

Antes de derivar conclusiones de los hallazgos del cuadro 5, es esencial destacar que la relación observada entre la densidad poblacional y los factores de la oferta puede ser en parte producto de situaciones que se confunden. Por ejemplo, el hecho de que los países con alta densidad poblacional hayan logrado en promedio niveles ligeramente más altos de desarrollo económico, y de que la mayoría de los países con alta densidad estén ubicados en Asia y Medio Oriente -mientras en general los de baja densidad son subsaharianos-, puede estar afectando nuestros resultados. Es necesario eliminar estos efectos para obtener una estimación más realista del papel de la densidad poblacional. Por ello se realizó un análisis de regresión para cada factor de la oferta, con base en los datos de 1989. La densidad poblacional, el nivel de desarrollo (medido por el logaritmo del PIB per cápita) y la región fueron las variables explicativas incluidas. ${ }^{11}$

${ }^{11}$ PIв per cápita en dólares ajustado a la paridad de acuerdo con el poder de compra (PNUD, 1991). 
GUADRO 4

Estimaciones de los factores de la oferta alimentaria para 90 países en desarrollo en 1962 y 1989, clasificadas por densidad poblacional alta, media y baja en 1989

\begin{tabular}{|c|c|c|c|c|}
\hline \multirow[b]{2}{*}{ Factor de la oferta } & \multicolumn{4}{|c|}{$\begin{array}{c}\text { Densidad poblacional en } 1989 \\
\text { (personas por ha de vierra } \\
\text { potencialmente cultivable) }\end{array}$} \\
\hline & $\begin{array}{r}\text { Alta } \\
(6.6)\end{array}$ & $\begin{array}{r}\text { Media } \\
(1.3)\end{array}$ & $\begin{array}{l}\text { Baja } \\
(0.3)\end{array}$ & $\begin{array}{l}\text { Total } \\
(2.7)\end{array}$ \\
\hline \multicolumn{5}{|l|}{1989} \\
\hline Proporción de tierra cultivada & 0.80 & 0.45 & 0.18 & 0.48 \\
\hline Frecuencia de cosecha & 1.01 & 0.70 & 0.48 & 0.73 \\
\hline \multicolumn{5}{|l|}{ Proporción de tierra para cultivos } \\
\hline alimenticios & 0.95 & 0.94 & 0.92 & 0.94 \\
\hline Rendimiento por cosecha ( $\mathrm{TGE}$ ) & 2.40 & 1.88 & 1.58 & 1.95 \\
\hline Multiplicador de comercio & 1.73 & 1.34 & 1.17 & 1.41 \\
\hline Proporción para consumo directo & 0.79 & 0.76 & 0.80 & 0.78 \\
\hline Multiplicador de producto animal & 1.12 & 1.11 & 1.14 & 1.12 \\
\hline Calorías diarias por persona & 2477 & 2467 & 2270 & 2405 \\
\hline \multicolumn{5}{|l|}{1962} \\
\hline Proporción de tierra cultivada & 0.68 & 0.38 & 0.14 & 0.40 \\
\hline Frecuencia de cosecha & 0.96 & 0.64 & 0.42 & 0.67 \\
\hline $\begin{array}{l}\text { Proporción de tierra para cultivos } \\
\text { alimenticios }\end{array}$ & \multicolumn{3}{|c|}{ Proporción de tierra para cultivos } & 0.92 \\
\hline Rendimiento por cosecha (TGE) & 1.98 & 1.22 & 1.35 & 1.52 \\
\hline Multiplicador de comercio & 1.11 & 1.01 & 1.01 & 1.04 \\
\hline Proporción para consumo directo & 0.82 & 0.74 & 0.77 & 0.78 \\
\hline Multiplicador de producto animal & 1.11 & 1.12 & 1.14 & 1.12 \\
\hline Calorías diarias por persona & 1998 & 2136 & 2173 & 2102 \\
\hline Número de países & 30 & 30 & 30 & 90 \\
\hline \multicolumn{5}{|l|}{ Tamaño de la población (millones) } \\
\hline 1989 & 2846 & 596 & 480 & 3922 \\
\hline 1962 & 1594 & 290 & 242 & 2127 \\
\hline
\end{tabular}

Los resultados de este ejercicio se resumen en el cuadro 5. Los fuertes efectos positivos de la densidad poblacional sobre la proporción de la tierra cultivada, frecuencia de cosecha y rendimiento por cosecha se confirman y son altamente significativos. La densidad no tiene un impacto significativo en los demás factores de la oferta o en el consumo calórico per cápita. Es probable que una explicación parcial de este último hallazgo sean los subsidios e intervención gubernamentales en la 


\section{CUADRO 5}

Resultados del análisis de regresión de determinantes de los factores de oferta alimentaria en 90 países en desarrollo, 1989

\begin{tabular}{|c|c|c|c|c|c|}
\hline \multirow[b]{3}{*}{ Factor de la oferta } & \multicolumn{5}{|c|}{ Variable explicativa ${ }^{\mathbf{a}}$} \\
\hline & \multirow[b]{2}{*}{$\begin{array}{l}\text { Densidad } \\
\text { poblacional }\end{array}$} & \multirow[b]{2}{*}{$\begin{array}{l}\text { PIB'per } \\
\text { cápita }\end{array}$} & \multicolumn{3}{|c|}{ Región $\mathrm{b}$} \\
\hline & & & $\begin{array}{l}\text { América } \\
\text { Latina }\end{array}$ & Asia & $\begin{array}{l}\text { Medio } \\
\text { Orientel } \\
\text { Norte de } \\
\text { Africa }\end{array}$ \\
\hline Proporción de tierra cultivada & +++ & & - & & \\
\hline Frecuencia de cosecha & +++ & & & + & \\
\hline $\begin{array}{l}\text { Proporción de tierra para cultiv } \\
\text { alimenticios }\end{array}$ & & & - & & \\
\hline Rendimiento por cosecha (TGE) & ++ & +++ & ++ & ++ & \\
\hline Multiplicador de comercio & & & & & +++ \\
\hline Proporción para consumo direc & & - & - & - & - \\
\hline Multiplicador de producto anim & & +++ & ++ & & \\
\hline Calorías diarias por persona & & +++ & & & +++ \\
\hline
\end{tabular}

$\mathrm{a}+++\mathrm{o}-\mathrm{-}: \mathrm{p}<0.01 ;++\mathrm{o}--: 0.01<\mathrm{p}<0.05 ;+\mathrm{o}-0.05<\mathrm{p}<0.1$.

b El grupo de referencia es África subsahariana.

agricultura, para prevenir aumentos en los precios al consumidor en países con severas restricciones en sus recursos naturales. ${ }^{12}$ En el cuadro 5 los resultados de la regresión indican efectos positivos altamente significativos del nivel de desarrollo en el rendimiento por cosecha, del multiplicador de producto animal y de las calorías diarias per cápita, así como un efecto negativo en la proporción directamente consumida. Estos hallazgos no son inesperados. A medida que aumentan los ingresos, los consumidores exigen y pueden pagar dietas de mayor cantidad y calidad. En consecuencia, aumentan también el consumo calórico por persona y el porcentaje de la dieta de origen animal. A medida que una mayor proporción de las cosechas se dedica al forraje y otros usos, disminuye la proporción consumida directamente por los humanos. Se supone que el efecto del nivel de desarrollo en el rendimiento de la cosecha es en gran medida consecuencia de los crecientes ingresos entre los agricultores, lo cual les permite

12 Véase en Alexandratos (1995) una exposición más amplia de la relación entre la razón tierra/persona y el consumo alimenticio per cápita. 
comprar mayores cantidades de insumos agrícolas: fertilizantes, pesticidas, agua, variedades mejoradas de semilla, maquinaria y técnicas agrícolas. Además, los países con ingresos más altos tienden a tener siperior infraestructura, mejorando así el acceso a los mercados y haciendo económicamente más atractivos los mayores rendimientos.

En varios casos los factores de la oferta también variaron de manera sistemática entre las distintas regiones (todos los efectos para América Latina, Asia y Medio Oriente se miden en relación con África subsahariana). En las tres regiones incluidas en el cuadro 5, la proporción de consumo humano directo fue menor que en África subsahariana, debido quizá a los bajos ingresos y a que en esta región rara vez se utilizan las cosechas para alimentar a los animales, dada su provisión relativamente alta de pastizales. Otros hallazgos importantes son que en $\mathrm{Me}$ dio Oriente existe mayor comercio y también un cosumo de calorías per cápita mayor; en América Latina se presentan niveles relativamente altos en el rendimiento por cosecha y en el multiplicador de producto animal, y en Asia los rendimientos por cosecha y la frecuencia de cosecha exceden significativamente los de África subsahariana.

\section{Adaptándose a la presión poblacional}

En las secciones anteriores se examinó, en primer lugar, la manera como el sistema de oferta de alimentos en los países en desarrollo respondía, en el tiempo, para hacer frente a la creciente demanda alimentaria, resultante sobre todo del rápido crecimiento poblacional entre 1962 y 1989. En segundo lugar, se compararon grupos de países con distintas densidades poblacionales en 1989. Ambas perspectivas pueden mostrar cómo las sociedades responden a la creciente presión poblacional; desafortunadamente, los resultados de las dos aproximaciones parecen inconsistentes. La perspectiva longitudinal de la gráfica 2 sugiere que la creciente demanda se cubrió, principalmente, por medio del aumento en el rendimiento y la expansión del comercio. Por el contrario, la perspectiva transversal de la gráfica 3 indica que una densidad más alta se compensa sobre todo usando mayores proporciones de tierra potencialmente cultivable y aumentando la frecuencia de cosecha. Esta aparente contradicción puede explicarse de la siguiente manera:

- El aumento en la densidad de población en los países en desarrollo, entre 1962 y 1989, ha sido en promedio mucho más pequeño que la diferencia en densidad entre los países más y menos densos. 
Entre 1962 y 1989 el tamaño de la población, y por lo tanto su densidad, aumentó $102 \%$ en promedio. En cambio, el grupo de países de alta densidad tuvo en promedio alrededor de 22 veces más personas por hectárea de tierra potencialmente cultivable que el grupo de países de menor densidad. Dado que una creciente densidad da lugar a aumentos en la proporción de tierra cultivada y en la frecuencia de cosecha (cuadro 5), no sorprende que a lo largo del tiempo los cambios en estos dos factores de la oferta sean menores que las diferencias entre países de alta y baja densidad.

- Entre 1962 y 1989 el PIB per cápita en los países en desarrollo aumentó $131 \%$ en promedio. Este aumento es mucho mayor que la diferencia de $29 \%$ entre los grupos de países con alta y baja densidad en $1989 .{ }^{13}$ Como el rendimiento por cosecha tiene una correlación positiva con el PIB per cápita, se esperaría encontrar un cambio mayor en el rendimiento, a lo largo del tiempo, que entre los niveles de densidad en 1989, como de hecho se observa en las gráficas 2 y 3.

Una vez que se consideran estas diferencias clave, las perspectivas longitudinal y transversal del vínculo entre la densidad poblacional y los factores de la oferta alimentaria son altamente consistentes entre sí. Esto se confirma con otros análisis de regresión, que fueron similares a aquellos mostrados en el cuadro 5, excepto que sejuntaron los datos de 1962 y 1989 y se introdujo para el tiempo una variable muda. Los coeficientes de regresión para la densidad no difirieron significativamente de los estimados en las regresiones de 1989, pero las variables mudas para el tiempo fueron significativamente distintas de cero en las regresiones del multiplicador de comercio y la proporción de tierra cultivada. ${ }^{14}$ Existen, por lo tanto, dos excepciones a la generalización de que los hallazgos longitudinales y transversales están en concordancia.

Primero, el multiplicador de comercio evidentemente está influido por variables distintas de las examinadas aquí. Los resultados de regresión del cuadro 5 no muestran ningún efecto estadístico de la densidad o del PIB per cápita sobre el comercio de alimentos; sin embargo, este comercio aumentó $35 \%$ entre 1962 y 1989 . Es probable

${ }^{13}$ Los Pir per cápita para los países de alta y baja densidad en 1989 fueron de 2603 y 2013 dólares, respectivamente. El PiB per cápita promedio aumentó de 990 en 1960 a 2285 dólares en 1989.

${ }^{14}$ En la regresión de la proporción de tierra cultivada el PIB per cápita se excluyó como variable explicativa porque no tuvo efecto significativo sobre la proporción cultivada en 1989 (cuadro 4) y está altamente correlacionada con el tiempo. 
que la liberalización del comercio en muchos países y la reducción de precios en el mercado mundial expliquen este hallazgo.

Segundo, la proporción de tierra cultivada aumentó entre 1962 y 1989 a un ritmo ligeramente inferior al que se hubiera esperado con base en el incremento de la densidad poblacional y en el efecto de la densidad sobre la proporción de tierra cultivada observado en 1989. Hay varias explicaciones posibles para este hallazgo:

- Los países más densamente poblados se han enfrentado con crecientes dificultades para aumentar la proporción de tierra cultivada porque se intensifica la competencia por el uso de la tierra potencialmente cultivable restante y disminuye su calidad relativa (más sobre este aspecto se verá posteriormente).

- La acelerada urbanización en los países en desarrollo ha tenido como consecuencia la concentración de la población en grandes ciudades. Esto puede haber llevado a una intensificación de la agricultura cerca de los centros de población más que a su expansión en zonas más remotas.

- El aumento en el comercio alimentario proveniente de los países más desarrollados ha reducido la presión para expandir las áreas de tierra cultivable.

- La creciente preocupación mundial sobre la necesidad de conservar los bosques y otros recursos naturales ha llevado a muchos gobiernos a establecer reservas naturales que están fuera de los límites de los campesinos.

Resumiendo, los cambios en los factores de la oferta alimentaria observados entre 1962 y 1989 y las diferencias entre los grupos de distinta densidad poblacional en 1989 son consecuencia de tres procesos: l') la creciente densidad poblacional ha tenido como resultado aumentos en la proporción de tierra cultivada, en la frecuencia de cosecha y en el rendimiento por cosecha; 2) el incremento en los ingresos ha llevado a aumentos en el rendimiento por cosecha, el multiplicador de producto animal y el consumo calórico, asî como también a disminuciones en la proporción de cultivos para el consumo directo; y 3) otras variables de variación temporal han expandido el comercio alimentario y limitado la expansión de la tierra cultivable.

\section{Acercándose a los límites del crecimiento}

El análisis anterior calculó las respuestas promedio de los factores de la oferta alimentaria ante la creciente presión poblacional. Ahora me 
propongo examinar cómo estas respuestas variaron según el grado de densidad poblacional; hay suficientes razones teóricas para creer que estas variaciones existen. Específicamente, a medida que aumenten su densidad poblacional y su demanda de alimento los países alcanzarán límites al crecimiento en algunos factores de la oferta. Por ejemplo, un país que ya haya explotado la mayor parte de su tierra potencialmente cultivable bajo producción de cultivos encontrará más dificultad para extender su área de tierra cultivable que otro, donde gran parte del área potencial esté aún sin utilizar. El rendimiento por cosecha y la frecuencia de cosecha no pueden aumentar indefinidamente. Así, se esperaría que los países con alta densidad, donde estos factores de la oferta ya muestran valores elevados, respondan de modo diferente ante el crecimiento poblacional que los que cuentan con baja densidad poblacional.

El cuadro 6 presenta el incremento porcentual en los factores de la oferta alimentaria entre 1962 y 1989 para cada uno de los tres grupos de densidad estudiados. La diferencia en los aumentos entre los de alta y baja densidad es más clara respecto al porcentaje de tierra cultivada ( 21 contra $37 \%$ ) y para el multiplicador de comercio (58 contra $17 \%$ ). Para la mayoría de los demás factores las diferencias son mucho más pequeñas y con frecuencia no monotónicas. Por ejemplo, el grupo de densidad media alcanzó el mayor aumento en rendimiento (59\%) y el de densidad baja el menor (25\%). La ausencia de un efecto monotónico sustancial del grado de densidad sobre el incremento en rendimiento por cosecha y frecuencia de cosecha resulta un poco inesperada, porque anteriormente se había observado que la densidad afectaba estos factores. Algunas explicaciones posibles para estos hallazgos son 1 ) no hay límites al crecimiento para estos factores; 2) dichos límites existen, pero los países aún están suficientemente lejos de ellos, de modo que desempeñan un papel mínimo por ahora; 3) los límites se acercan, aunque este efecto no es visible en la evidencia empírica debido a factores compensatorios (por ejemplo, aumentar el rendimiento en cierta medida es más difícil cuando ya es alto, pero los subsidios gubernamentales pueden compensar este efecto); 4) hay errores en los datos de la FAo. Lo más probable es que tal efecto sea consecuencia de una combinación de las últimas tres explicaciones.

Como resultado de los cambios en los factores de la oferta en el periodo de 27 años comprendido entre 1961 y 1963, y 1988 y 1990, la oferta alimentaria total en los 90 países aumentó 138\%. En los grupos 
CUADRO 6

Incremento porcentual en factores de la oferta alimentaria, tamaño de la población y consumo de aủimentos por persona entre 1962 y 1989 para 90 países en desarrollo clasificados de acuerdo con su densidad poblacional (promedios no ponderados)

\begin{tabular}{lrrrr}
\hline & \multicolumn{4}{c}{ Densidad poblacional en 1989 } \\
\cline { 2 - 5 } & Alta & Media & Baja & Total \\
& $(6.6)$ & $(1.3)$ & $(0.3)$ & $(2.7)$ \\
\hline Factor de la oferta & & & & \\
Proporción de tierra cultivada & 21.2 & 21.0 & 37.2 & 28.5 \\
Frecuencia de cosecha & 14.8 & 15.3 & 23.2 & 17.7 \\
Proporción de área para cultivos & & & & \\
$\quad$ alimenticios & 2.1 & 1.7 & 3.5 & 2.4 \\
Rendimiento por cosecha (TGE) & 38.0 & 58.8 & 24.9 & 40.6 \\
Multiplicador de comercio & 57.5 & 29.8 & 17.4 & 34.9 \\
Proporción para consumo directo & -2.2 & 4.0 & 3.1 & 1.6 \\
Multiplicador de producto animal & 1.1 & -0.6 & 0.0 & 0.2 \\
Población & 92.3 & 109.3 & 103.5 & 101.7 \\
Consumo de alimentos per cápita & 24.7 & 16.5 & 5.0 & 15.4 \\
Consumo total de alimentos & 143.5 & 149.5 & 120.6 & 137.9 \\
\hline
\end{tabular}

Nota: El cambio porcentual promedio no es igual al cambio en promedio de los factores de la oferta.

de densidad alta y media se incrementó a una tasa ligeramente más alta ( 144 y $150 \%$, respectivamente), pero en el grupo de baja densidad aumentó sólo 121 por ciento.

\section{Perspectivas para el futuro}

La demanda de cultivos en los países en desarrollo crecerá de manera sustancial durante las próximas décadas, antes de que supuestamente se estabilice, lo cual sucederá cuando las tasas de crecimiento de la población y del consumo alimentario por persona se acerquen a cero, tal como se espera para mediados del próximo siglo. Varios son los factores que contribuirán a este crecimiento. Primero, de acuerdo con las últimas proyecciones de Naciones Unidas (1995), se proyecta que el tamaño de la población de los 90 países incluidos en este estudio crecerá más del doble, llegando a 8600 millones de habitantes en el año 2050. Segundo, es probable que el consumo calórico siga au- 
mentando. Alexandratos (1995) proyecta que el consumo calórico promedio en 2025 podría llegar a 2900 calorías diarias por persona (de las 2472 que alcanzó en 1989). ${ }^{15}$ Para 2050 este promedio podría ser aún mayor, aunque probablemente permanecería por debajo de las 3400 calorías por persona que se consumen en los países desarrollados. Tercero, a pesar de la casi constancia del multiplicador de producto animal entre 1962 y 1989 , es probable que los crecientes ingresos lleven a los consumidores a exigir que su dieta tenga mayor proporción de productos animales que la que tiene ahora. ${ }^{16}$ Si eso pasara, la proporción de cultivos para el consumo humano directo tendría que disminuir para permitir que los cultivos se usaran como forraje. De hecho, como la conversión de forraje en productos animales es relativamente ineficiente en términos energéticos, cualquier incremento en el multiplicador de producto animal tendría que compensarse con una reducción más que correspondiente de la proporción de consumo directo. El resultado neto de los aumentos de $112 \%$ en la población, $30 \%$ en el consumo calórico diario por persona, y $10 \%$ en el uso interno de las cosechas, con un cambio hacia un consumo mayor de productos animales, ${ }^{17}$ sería un aumento total del uso interno de las cosechas comestibles en los países en desarrollo de cerca de $200 \%$ durante el periodo de 60 años comprendido entre 1989 y 2050 , y más de la mitad de tal incremento sería consecuencia del crecimiento poblacional. Esta expansión de la oferta alimentaria agregada sería más grande que la lograda entre 1962 y 1989 (134\%), aunque la tasa anual del incremento futuro resultara sustancialmente más baja que en el pasado ( 1.8 contra $3.1 \%$ anual).

Satisfacer este gran aumento en la demanda implicará sin duda incrementos en cuatro de los cinco factores que determinan la disponibilidad interna de cultivos alimenticios, es decir, proporción de tierra cultivada, frecuencia de cosecha, rendimiento por cosecha y comercio alimentario (el quinto factor, la proporción de área dedicada

\footnotetext{
${ }^{15}$ Este cálculo de Alexandratos (1995) está basado en la suposición de que en 2025 ningún país tendrá un consumo calórico menor a 2700.

${ }^{16}$ La casi constancia del coeficiente de producto animal no significa que el consumo de productos animales por pesona haya permanecido constante, porque el consumo alimenticio total por persona aumentó $15 \%$ entre 1962 y 1989. En los países donde el coeficiente de producto animal permaneció constante, el consumo de productos animales cambió al mismo ritmo que el consumo alimenticio total. 2050 .

${ }^{17}$ Es decir, se supone que el producto de $\mathrm{F}_{\mathrm{d}}$ y $\mathrm{F}_{\mathrm{a}}$ aumentará $10 \%$ entre $1989 \mathrm{y}$
} 
a cultivos alimenticios, está ahora en 0.94: es probable que cualquier aumento futuro sea pequeño). Hay muchas combinaciones de variaciones en los factores que, en teoría, podrían conjuntamente triplicar el uso interno de las cosechas. Aquí no se hará intento alguno de predecir cómo cambiará de hecho cada factor de la oferta a lo largo del tiempo, sino que, sobre todo para efectos ilustrativos, se describirá brevemente un conjunto de los posibles niveles de los factores (promedios ponderados) en 2050. Los cambios en este conjunto de factores se pueden advertir en el cuadro 7 .

CUADRO 7

Cambios en los factores

\begin{tabular}{lccc}
\hline & 1989 & 2050 & Aumento \\
& Real & Hipotético & porcentual \\
\hline Factor de la oferta & & & \\
Proporción de tierra cultivada & 0.32 & 0.38 & 20 \\
Frecuencia de cosecha & 0.87 & 1.04 & 20 \\
Rendimiento por cosecha (TGE) & 2.24 & 4.48 & 100 \\
Multiplicador de comercio & 1.03 & 1.08 & 5 \\
Cambio total en oferta alimentaria & & & 202 \\
\hline
\end{tabular}

En este escenario hipotético la contribución más grande se lograría acrecentando los rendimientos promedio $(+100 \%) .{ }^{18}$ Esto implica una tasa anual de aumento en los rendimientos durante las siguientes seis décadas, que equivaldría a sólo cerca de la mitad de lo que se logró entre 1962 y 1989 ( 1.1 contra $2.1 \%$ anual). El rendimiento promedio en 2050 sería por lo tanto de 4.5 toneladas por hectárea, que ya han logrado obtener varios países en desarrollo (FAO, 1990).

También harían una contribución sustancial los incrementos futuros en la proporción de tierra potencialmente cultivable utilizada (de 0.32 a 0.38 ). Lograr esto requeriría transferir 170 millones de hectáreas de tierra de su uso actual -gran parte son selvas- a suelo agrícola (se calcula que el área total de selvas y bosques en los países en desarrollo en 1989 es de 2200 millones de hectáreas; FAo, 1990).

${ }^{18}$ La mezcla de cultivos sin duda cambiará entre 1989 y 2050 , y las estimaciones usadas en esta ilustración representan por lo tanto promedios ponderados de los rendimientos de distintos cultivos. 
La proporción promedio de tierra potencialmente cultivable utilizada (0.38) en 2050 aún estaría bastante por debajo de la proporción (0.8) observada en los países con mayor densidad poblacional en 1989. Los incrementos futuros en la oferta alimentaria también provendrían de una mayor frecuencia de cosecha (un alza de $20 \%$ : de 0.87 a 1.04 entre 1989 y 2050), alcanzando un nivel similar al que tienen ahora los países con alta densidad poblacional. Finalmente, una pequeña contribución provendría de un aumento en el multiplicador de comercio $(+5 \%)$. Aunque modesto en tamaño, este cambio implica que se muliplicaría varias veces el comercio de los países desarrollados a los países en desarrollo, porque la base a la cual se aplica el multiplicador también se está expandiendo. De manera conjunta, los aumentos hipotéticos en estos cuatro factores bastarían para triplicar la oferta alimentaria interna para el año 2050.

\section{Conclusión}

Aunque cualquier proyección para mediados del próximo siglo debe verse como especulativa, en general el escenario anterior para 2050 parece factible. Las recientes proyecciones en el corto plazo -para 2010 o 2020- hechas por el Banco Mundial (Mitchell e Ingco, 1995); la Fao (Alexandratos, 1995) y el Instituto Internacional de Investigación sobre Políticas Alimentarias (Agcaoili y Rosegrant, 1995), con base en modelos econométricos, tampoco prevén obstáculos importantes a la expansión continua de la oferta alimentaria. Es por lo tanto probable que se puedan evitar serias y persistentes carencias alimentarias en el ámbito global si los gobiernos emprenden con determinación los esfuerzos para mejorar las políticas económicas y facilitar la divulgación de nueva tecnología y la inversión en investigación y recursos humanos.

Sin embargo, aunque la situación agregada parece manejable, varias cuestiones críticas requieren una atención urgente. Primero, la expansión de la producción de alimentos ha sido responsable de gran parte de la degradación de los recursos ambientales. La creciente demanda de tierra agrícola es una de las principales causas de la deforestación y de la pérdida de la biodiversidad. Prácticas agrícolas poco adecuadas han llevado a la pérdida de suelo, sobreexplotación de recursos hidrológicos limitados y contaminación por uso de fertilizantes y pesticidas. Además, la agricultura es responsable de una pro- 
porción significativa de la emisión de gases de invernadero (por ejemplo, el bióxido de carbono proveniente de la deforestación, el uso de combustibles para maquinaria y transportes, el metano de los arrozales y de la digestión del ganado, el óxido nitroso del fertlizante). En la práctica será inevitable cierto cambio ambiental en vista de la necesidad de expandir la producción de alimentos, aunque se puede y debe hacer mucho más por minimizar el impacto ambiental adverso y la degradación de los recursos agrícolas. Evitar un mayor deterioro ambiental será costoso y por lo tanto provocará un alza en los precios de los alimentos. Como consecuencia del constante aumento de las restricciones ambientales y de la creciente demanda, es poco probable que los precios de los alimentos en el mercado mundial continúen su disminución pasada y de hecho, podrían aumentar de manera significativa durante las próximas décadas. ${ }^{19}$

Segundo, en los países en desarrollo cerca de 800 millones de personas aún sufren desnutrición, y se espera que este total disminuya muy lentamente a lo largo del tiempo (FAO y oms, 1992). Dado que los precios mundiales de los alimentos se redujeron en el pasado, es evidente que la desnutrición no es resultado principalmente de una escasez global de alimentos. ${ }^{20}$ Por el contrario, los pobres carecen del ingreso para adquirir las reservas de alimento disponibles (Sen, 1981). Reducir la desnutrición requiere por lo tanto un esfuerzo concertado para eliminar la pobreza.

Tercero, los problemas para satisfacer la creciente demanda de alimentos serán mayores en los países más densamente poblados. Los 30 países que cuentan con la densidad poblacional más alta (incluyendo India, China, Indonesia, Bangladesh y Pakistán) reúnen $72 \%$ de la población de los países en desarrollo. Para dicho grupo la proporción de tierra cultivada ya es de $80 \%$, y será imposible aumentarla $20 \%$, como supone en promedio el escenario anterior. La demanda futura de alimentos en estos países tendrá que ser satisfecha, por lo tanto, con incrementos aún mayores en el rendimiento por cosecha,

\footnotetext{
${ }^{19}$ En el pasado las políticas proteccionistas de la Unión Europea y Estados Unidos han resultado en excedentes en algunos cultivos, deprimiendo los precios internacionales de los alimentos. Se espera que los efectos de estas políticas disminuyan en el futuro; esto agregará presión adicional al alza de los precios en el mercado mundial.

${ }^{20}$ La desnutrición y la poca disponibilidad de alimentos están más asociadas en países que dependen ampliamente de la productividad de su propia agricultura para la oferta de alimentos, ingresos y empleo. En tales contextos, aumentar la producción agrícola es necesariamente la clave para reducir la pobreza.
} 
frecuencia de cosecha y multiplicador de comercio. Una consecuencia probable es que los países densamente poblados importen más alimentos, no sólo de los países desarrollados, sino también de otros países en desarrollo con amplias reservas de tierra potencialmente cultivable (por ejemplo los de América Latina). Suponiendo que se eliminen las barreras comerciales y se disminuya el costo del transporte, los alimentos se producirán cada vez más en países y regiones que ten gan los costos más bajos de producción alimentaria. La importación de alimentos será difícil para los países más pobres, y muchos tratarán de seguir dependiendo de la ayuda humanitaria. ${ }^{21}$ Esta ayuda puede ser más difícil de conseguir si en el futuro las reservas mundiales de cereales permanecen bajas.

Muchas de las discusiones anteriores sobre el problema alimentario global se han centrado en los medios disponibles para expandir la oferta de productos agrícolas. Se ha prestado menos atención a las medidas de la demanda, aunque podrían hacer contribuciones sustanciales. Por ejemplo, las dietas de algunos países desarrollados ya contienen una proporción de productos animales tan alta que podría ser perjudicial para la salud. En varios de ellos se han emprendido esfuerzos por reducir esta proporción, y deben ser fortalecidos. Sin embargo, la forma más importante en que podría amortiguarse la demanda futura es deteniendo aún más el crecimiento poblacional en los países en desarrollo. Hay cada vez mayor disponibilidad de medios deseables y efectivos para reducir la fecundidad humana (Bongaarts, 1994) que deberían recibir mayor prioridad.

\section{Bibliografía}

Agcaoili, Mercedita y Mark W. Rosegrant (1995), "Global and Regional Food Supply, Demand, and Trade Prospects to 2010", en Nurul Islam (ed.), Population and Food in the Early Twenty-First Century: Meeting Future Food Demand of an Increasing Population, Washington D.C., International Food Policy Research Institute, pp. 61-83.

Alexandratos, Nikos (ed.) (1995), World Agriculture: Towards 2010: A FAo Study, Nueva York, John Wiley \& Sons [publicado en francés por Polytechnia (París) y en español por Mundi-Prensa, Madrid y México].

${ }^{21}$ En países con monedas devaluadas la compra de alimento en el mercado mundial se volverá más costosa, dificultando más su dependencia de importaciones de alimentos. 
Banco Mundial (1992), Price Prospectsıfor Major Primary Commodities, Washington D. C.

Bongaarts, John (1994), "Population Policy Options in the Developing World", Science, núm. 263, pp. 771-776.

Brown, Lester R. y Hal Kane (1994), Full House: Reassessing the Earth's Population Carrying Capacity, Nueva York, W. W. Norton.

Cohen, Joel E. (1995), How Many People Can the Earth Support?, Nueva York, W. W. Norton.

Dyson, Tim (1996), Population and Food: Global Trends and Future Prospects, Londres-Nueva York, Routledge.

Ehrlich, Paul R. y Anne H. Ehrlich (1990), The Population Explosion, Nueva York, Simon \& Schuster.

_ tion, and Environment", Population and Development Review, vol. 19, núm. 1, pp. 1-32.

fAo (Food and Agriculture Organization) (1990), fAo Production Yearbook, vol. 44, Roma.

(1992), Agrostat. PC: Computerized Information Series, Roma.

- y oms (1992), Nutrition and Development: A Global Assessment, Roma.

Heilig, Gerhard K. (1994), "How Many People can be Fed on Earth?", en Wolfgang Lutz (ed.), The Future Population of the World: What can We Assume Today?, Londres, Earthscan Publications for the International Institute for Applied Systems Analysis, pp. 207-261.

Johnson, D. Gale (1984), "World Food and Agriculture", en Julian L. Simon y Herman Kahn (eds.), The Resourceful Earth: A Response to Global 2000, Oxford-Nueva York, Basil Blackwell, pp. 67-112.

Malthus, Thomas (1959), Population: The First Essay, Ann Arbor, University of Michigan Press (Ann Arbor Paperbacks) [1798].

Mitchell, Donald O. y Merlinda D. Ingco (1995), "Global and Regional Food Demand and Supply Prospects", en Nurul Islam (ed.), Population and Food in the Early Twenty-First Century: Meeting Future Food Demand of an Increasing Population, Washington D. C., International Food Policy Research Institute, pp. 49-60.

Naciones Unidas (1995), World Population Prospects: The 1994 Revision, Nueva York. PNUD (Programa de las Naciones Unidas para el Desarrollo) (1991), Human Development Report 1991, Nueva York, Oxford University Press.

Sen, Amartya K. (1981), Poverty and Famines: An Essay on Entitlement and Deprivation, Oxford, Oxford University Press.

Simon, Julian L. (1981), The Ultimate Resource, Princeton, Princeton University Press.

Smil, Vaclav (1987), Energy, Food, Environment, Oxford, Clarendon Press. (1994), "How Many People can the Earth Feed?", Population and Development Revieu, vol. 20, núm. 2, pp. 255-292. 
